\title{
A Review of Cognitive Therapy for Mental Disorders Based on Bias Modification
}

\section{Shirin Khodadadi Arkavini' ${ }^{1}$, Mojtaba Khodadadi ${ }^{*}$, Hosein Davoodi ${ }^{3}$}

${ }^{1}$ Department of Counseling, Arak Branch, Islamic Azad University, Arak, Iran

${ }^{2}$ Department of Baisic Sciences, Emam Hossein University, Tehran, Iran

${ }^{3}$ Department of Counseling and Psychology, Khomein Branch, Islamic Azad University, Khomein, Iran

\section{ABSTRACT}

Introduction: Cognitive bias modification procedures are considered therapist-independent, computer-based, and relatively new interventions, which are mainly used as outpatient therapies for anxiety, emotional disorders, and different types of addiction. This paper aimed to present the latest findings of the studies on cognitive bias modification to enhance the knowledge of experts and researchers of the effects of such procedures in both practice and theory. At first, cognitive bias modification was briefly defined. Then, its different procedures (attention bias modification, interpretation bias modification, and memory bias modification) and the therapies based on these modification procedures were introduced. Then, the studies conducted on the effectiveness of such therapies were reviewed and their results were discussed. Conclusion: All the studies suggested, in a relatively consistent and significant way, that it is possible to modify cognitive bias processes in various disorders, such as anxiety and emotional disorders, mood disorders, chronic pain, obsessive disorders, addiction, and intellectual rumination. Furthermore, computer programs for cognitive bias modification and dot-probe tasks are suitable alternatives or complementary options for reducing or treating clinical symptoms and cognitive biases. In addition to the need for inventing therapy applications and adapting cultural characteristics of English versions for the Iranian population, new investigations are required to evaluate both the clinical crowd and the effectiveness of these modification procedures for various disorders.

\section{Keywords: \\ 1. Cognition \\ 2. Psychology \\ 3. Bias \\ 4. Outpatients}

*Corresponding Author: Mojtaba Khodadadi

Email:kh@sinapsycho.com 


\title{
مرورى بر درمان شناختى اختلالات روانى مبتنى بر اصلاح سوگيرى
}

\author{
شيرين خدادادى اركوينى'، مجتبى خدادادى "ّ"، حسين داوودى"

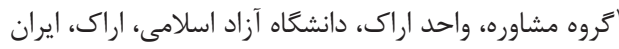

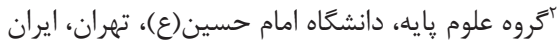

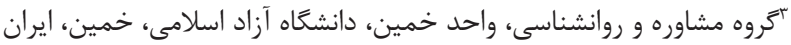

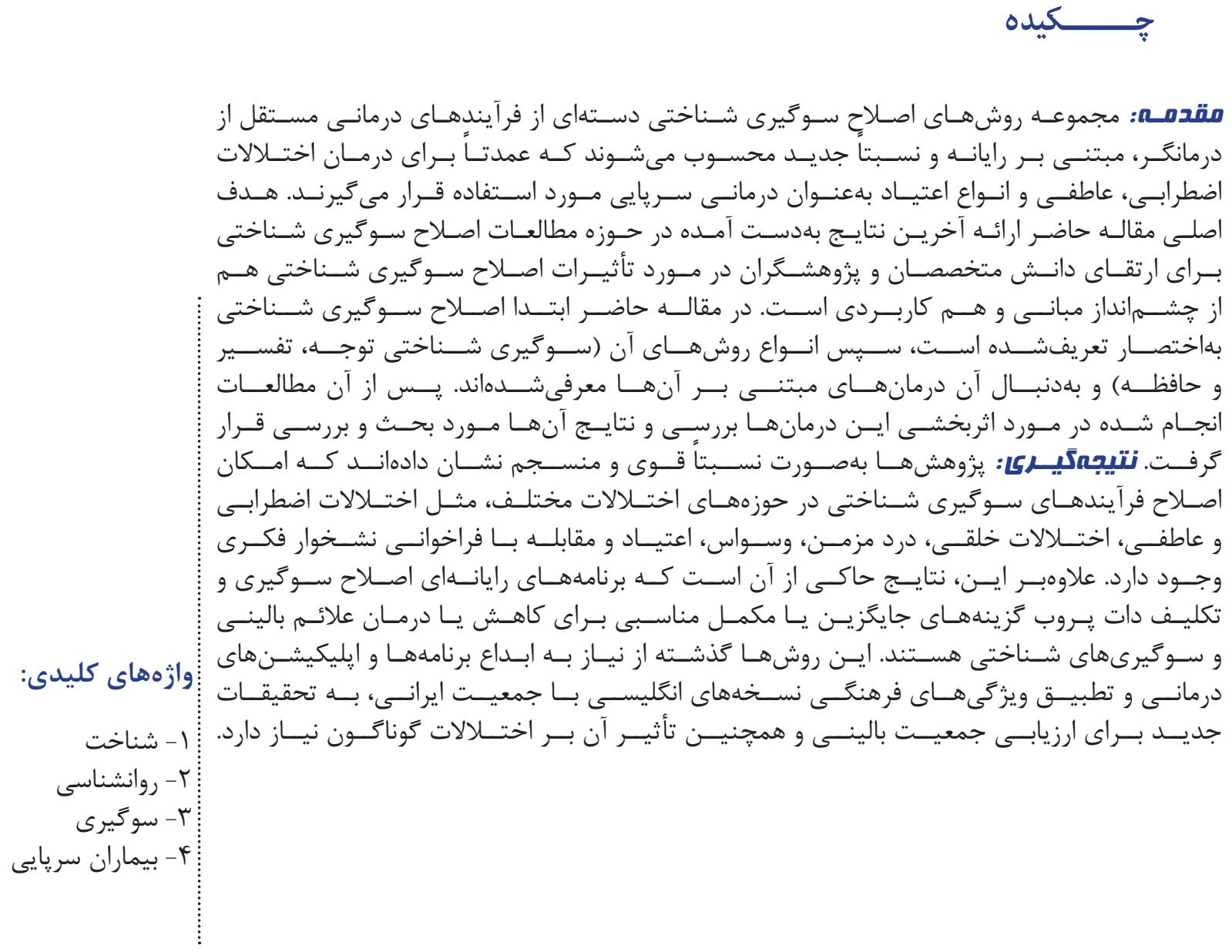

*ونيسنده مسئول: مجتبى خدادادى 


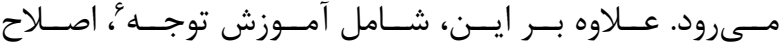

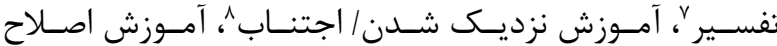

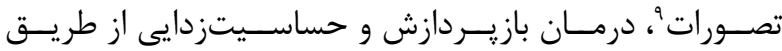

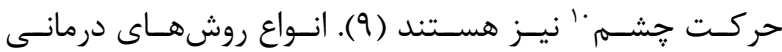

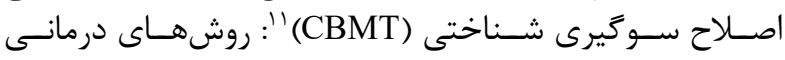

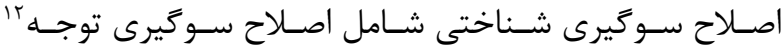

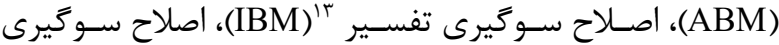

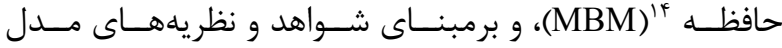

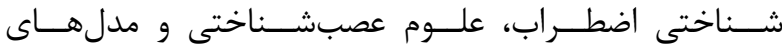

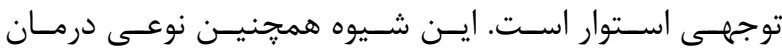

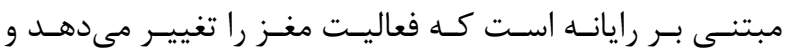

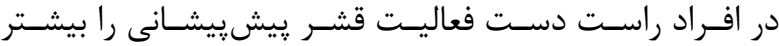

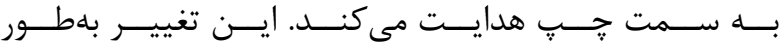

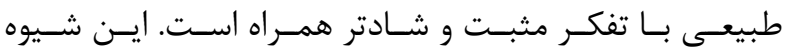

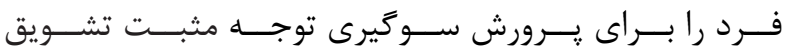

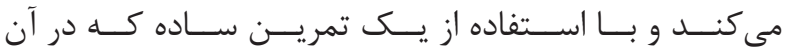

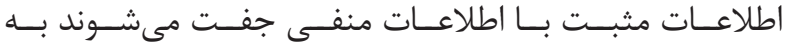

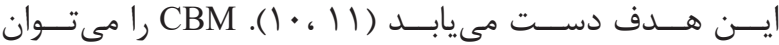

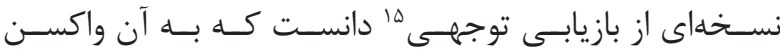

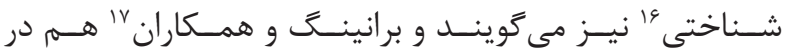

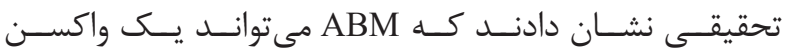

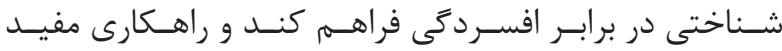

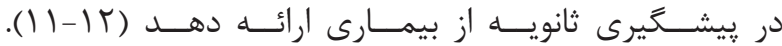

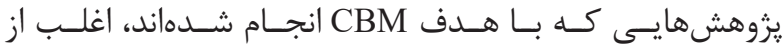

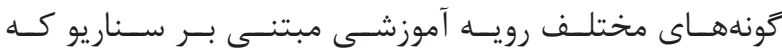

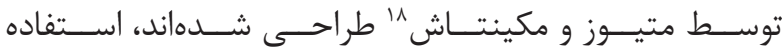

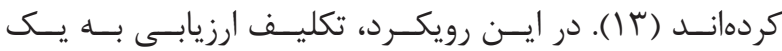

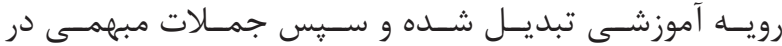

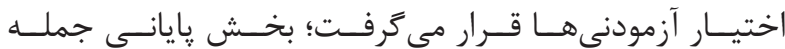

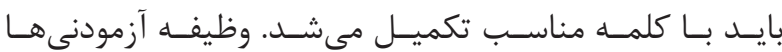

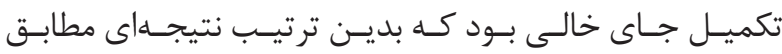

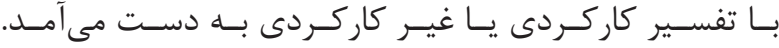

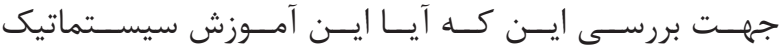

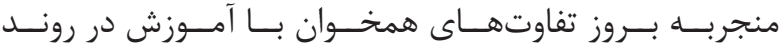

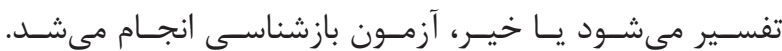

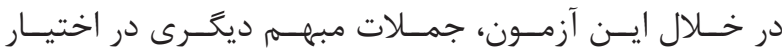

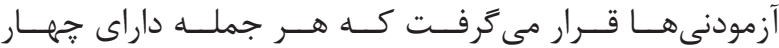

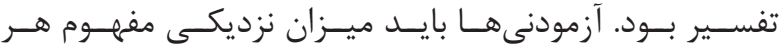

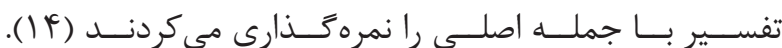

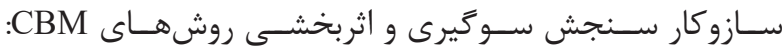

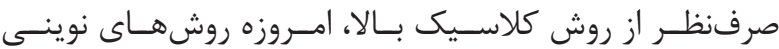

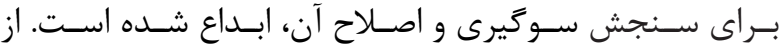

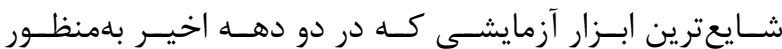

\section{${ }^{1}$ Cognitive Bias}

${ }^{2}$ Cognitive Bias Modification

${ }^{3}$ Implicit

${ }^{4}$ Explicit

${ }^{5}$ Post-traumatic Stress Disorder (PTSD)

${ }^{6}$ Attention Training

${ }^{7}$ Interpretation Modification

${ }^{8}$ Approach/avoid Training

${ }^{9}$ Imagery Modification Training

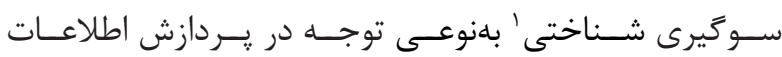

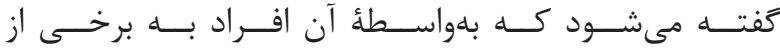

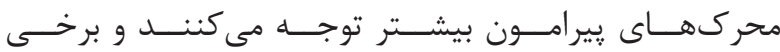

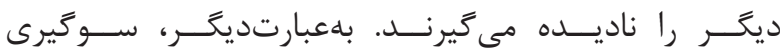

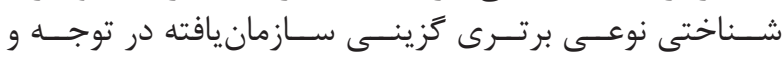

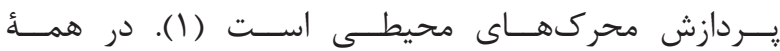

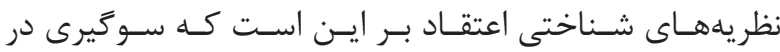

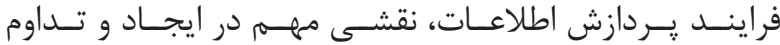

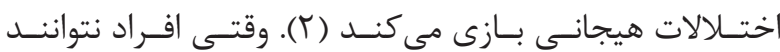

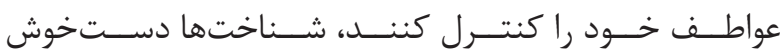

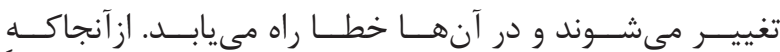

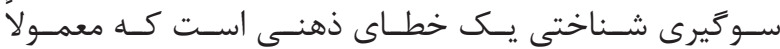

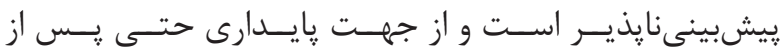

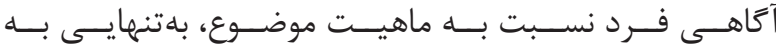

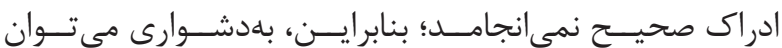

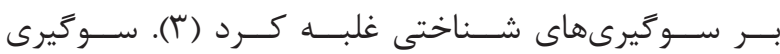

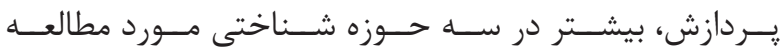

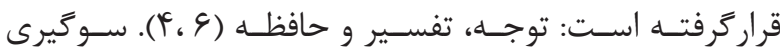

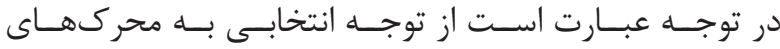

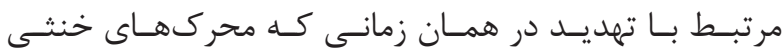

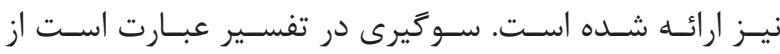

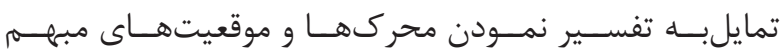

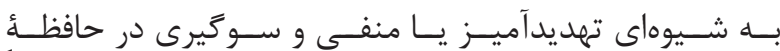

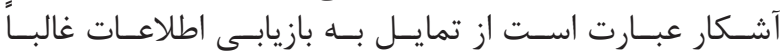

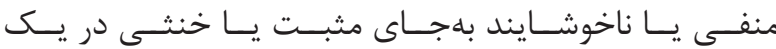

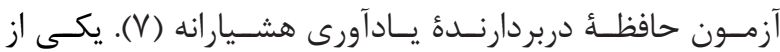

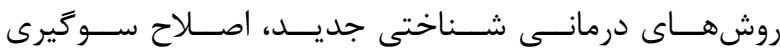

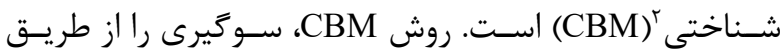

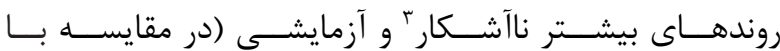

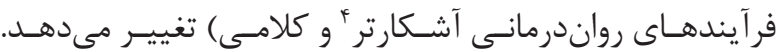

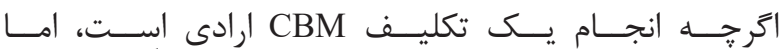

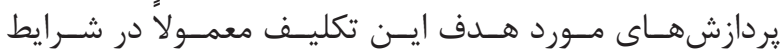

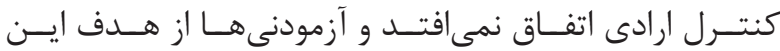

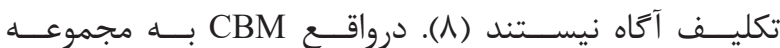

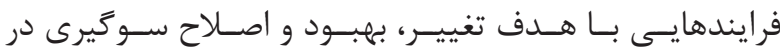

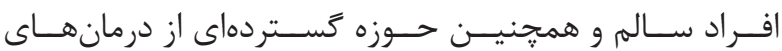

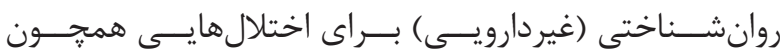

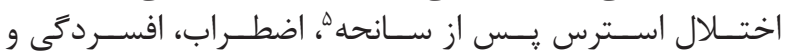

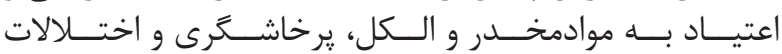

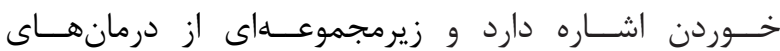

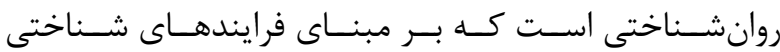

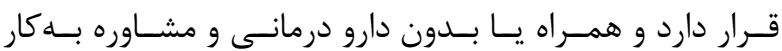

${ }^{10}$ Eye Movement Desensitization Reprocessing Therapy ${ }^{11}$ Cognitive Bias Modification Therapy

${ }^{12}$ Attention Bias Modification

${ }^{13}$ Interpretation Bias Modification

${ }^{14}$ Memory Bias Modification

${ }^{15}$ Attentional retaining

${ }^{16}$ Cognitive Vaccine

${ }^{17}$ Browing

${ }^{18}$ Mathews \& Mackintosh 


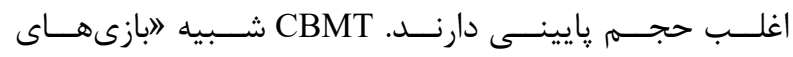

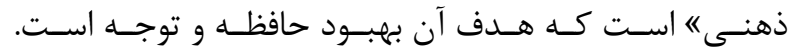

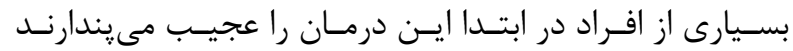

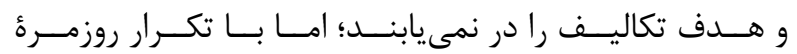

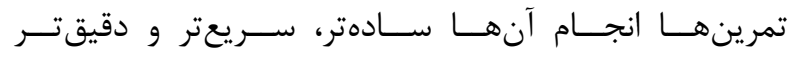

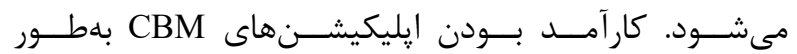

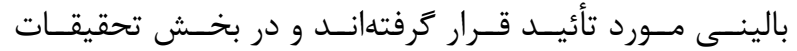

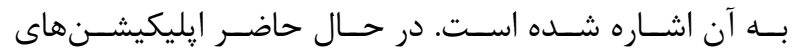

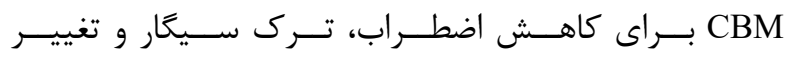

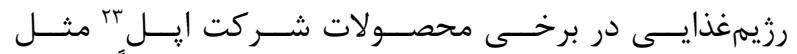
Appstore

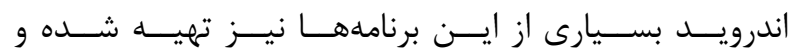

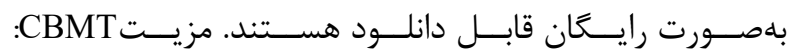

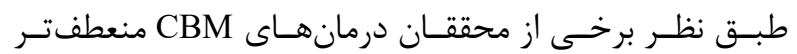

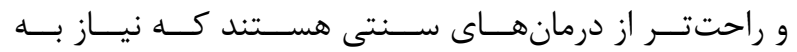

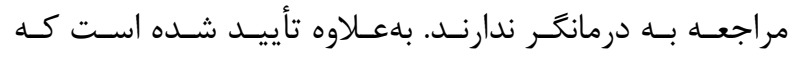

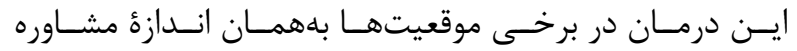

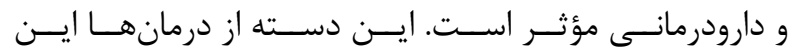

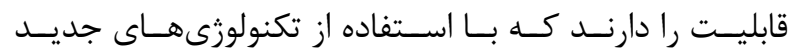

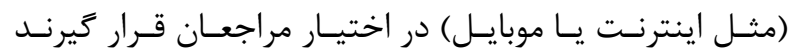

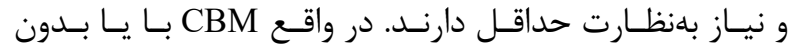

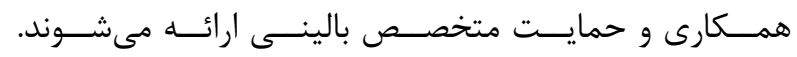

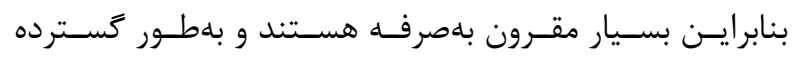

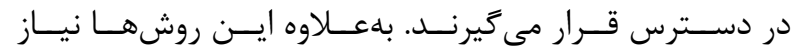

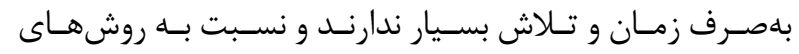

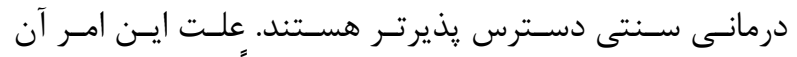

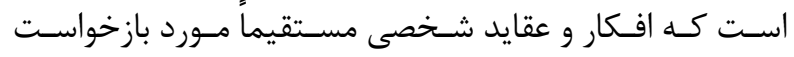

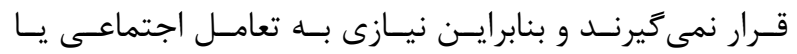

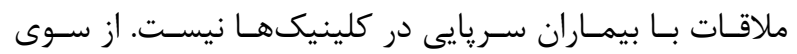

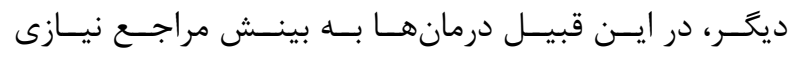

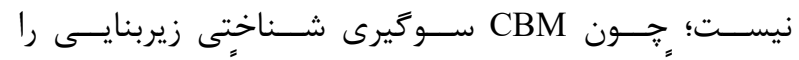

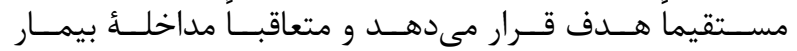

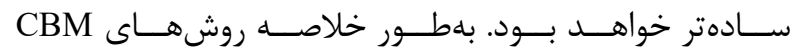

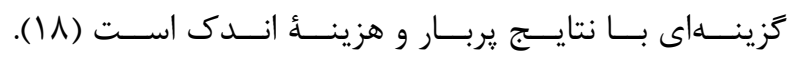

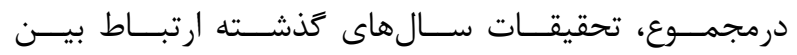

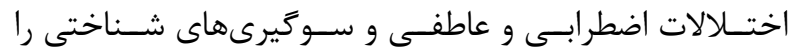

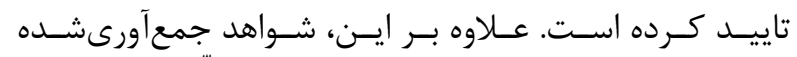

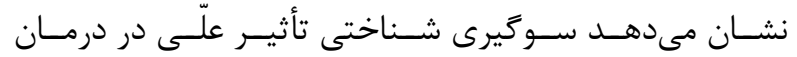

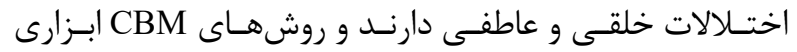

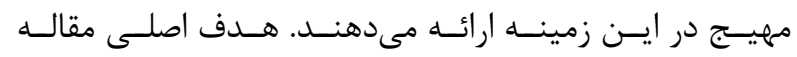

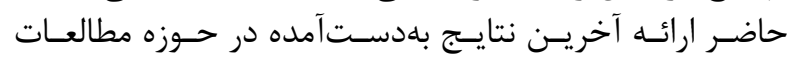

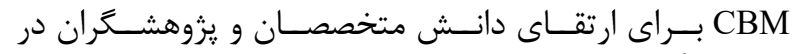

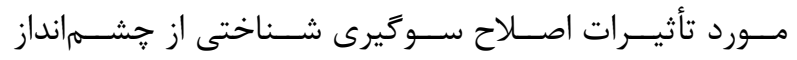

\section{${ }^{19}$ Stroop}

${ }^{20}$ Dot probe

${ }^{21}$ MacLeod

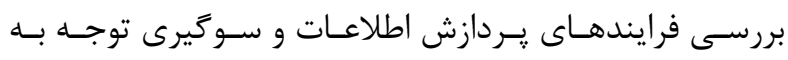

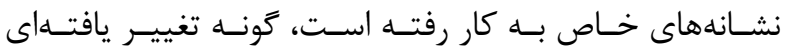

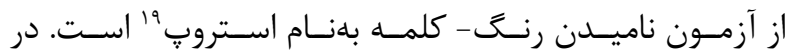

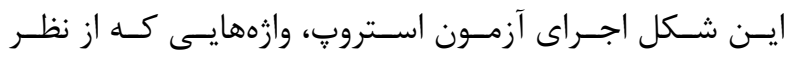

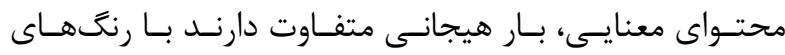

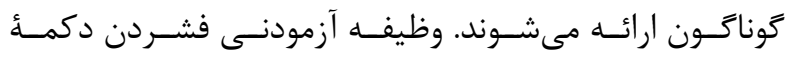

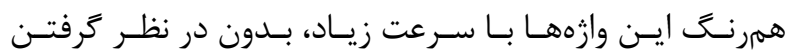

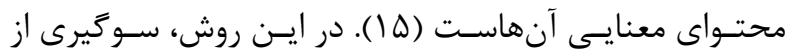

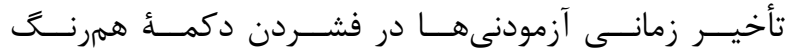

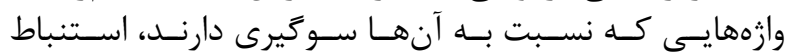

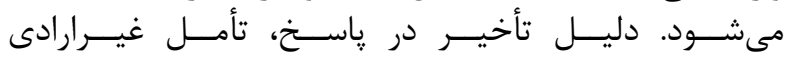

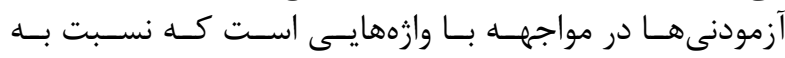

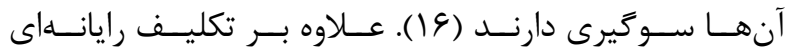

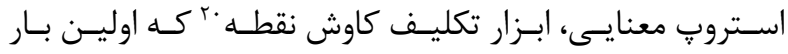

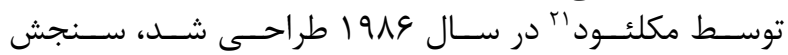

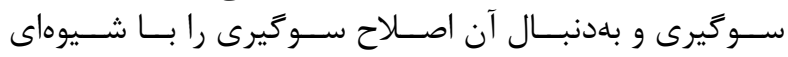

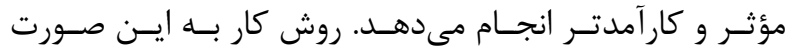

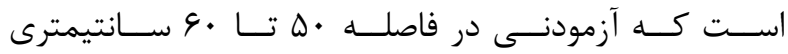

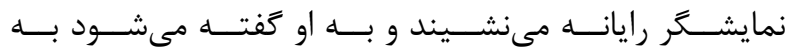

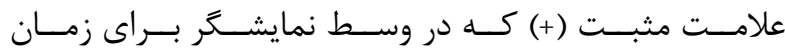

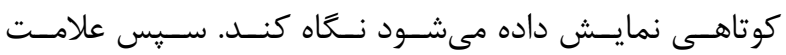

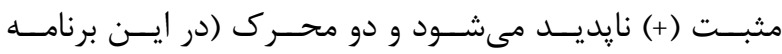

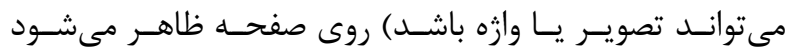

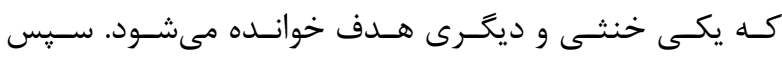

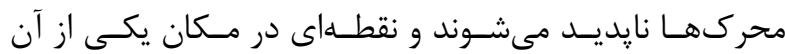

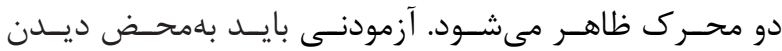

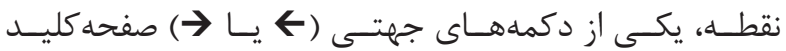

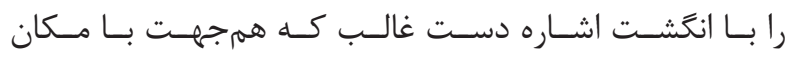

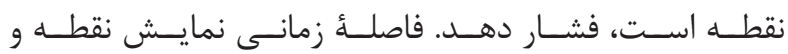

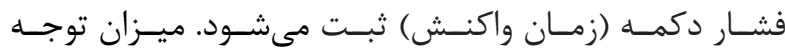

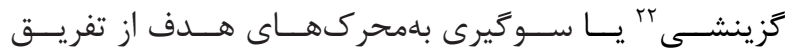

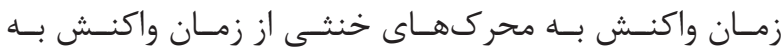

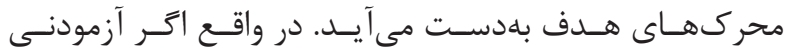

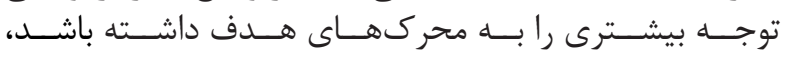

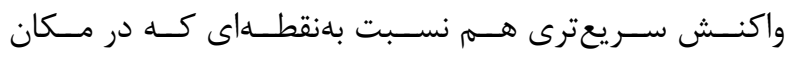

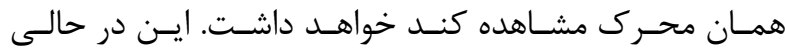

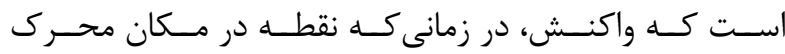

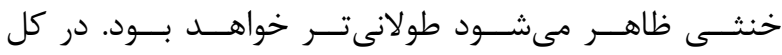

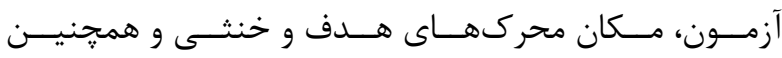

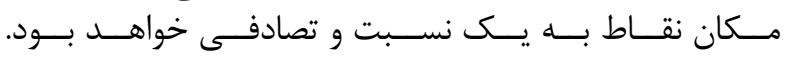

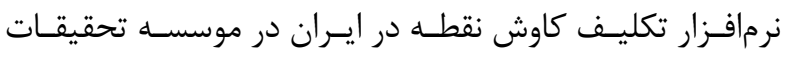

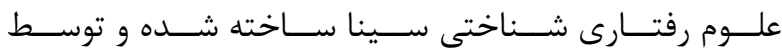

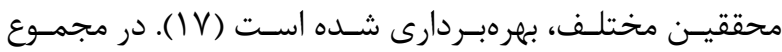

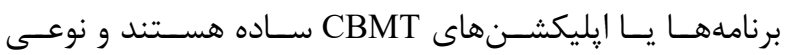

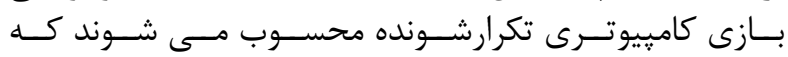

${ }^{22}$ Selective attention

${ }^{23}$ Apple 


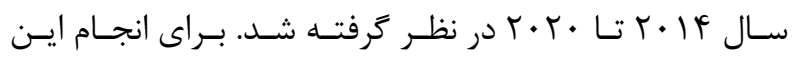

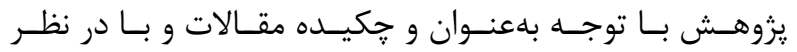

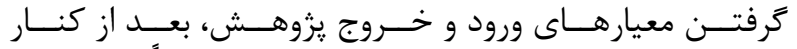

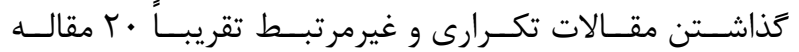

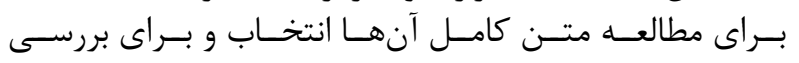

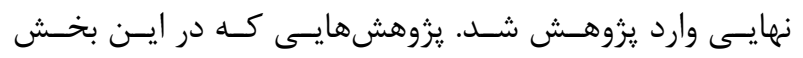

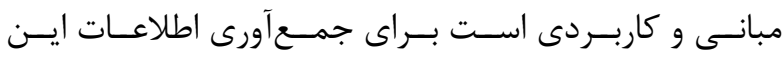

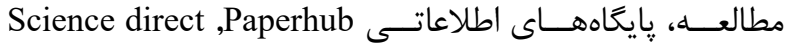
Pubmed, APA ,SID ,Google scholar,Sivilica

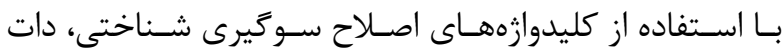

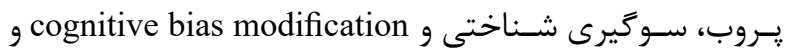

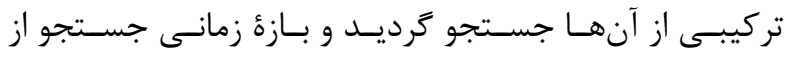

\begin{tabular}{|c|c|c|c|c|}
\hline خلاصه ثتايج & ثوع تحقيق & سال اجرا & محققان & رديف \\
\hline 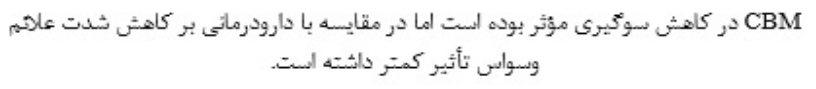 & تحفيق مداخلهاى با طرمن بسآزمون & $r \cdot 19$ & محسن يوريان و همكاران (19) مج) & 1 \\
\hline در كاهش علانمت افسردكى و بهبود (سوكيرى تفسيرى، نكرش ناكارآمد و حافظه) موثر & 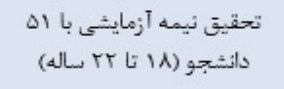 & $r \cdot 11$ & 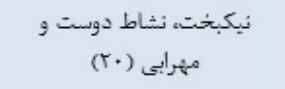 & r \\
\hline CBM بر كاثش وابستكى بـه سيكار موثر است. & 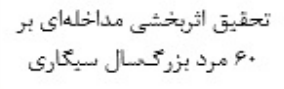 & $r \cdot 1 \mathrm{r}$ & 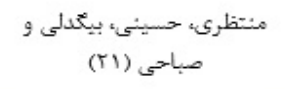 & $r$ \\
\hline نويد بخش مداخلهاى جايكزين يا مكمل در كاعش اضطراب اجتماعى و سوكيرى است. CBM & ساخت و اعتباريابى & $r \cdot 1 \mathrm{r}$ & شفيعى، زاع و علىيور (TT) & f \\
\hline اصلاح سوكيرى توجه در تشخيص و درمان اختلال اضطراب فراكير و بدريخت انتارى مؤتر & 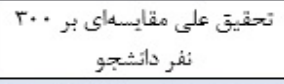 & $r \cdot 14$ & 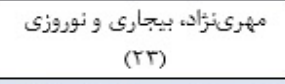 & $\Delta$ \\
\hline CBM در بيشغيرى و درمان جاقىى مؤثر است. CBM & تحقيق آزمايشى با هr فرد جاق & $r \cdot 19$ & تقوى، طاوسى و كودرزى (Tf) & 9 \\
\hline CBMT بر كاهش و شدت درد، اضطراب، الفسردكىى و استرس مؤثر است. & تحقيق آزمايشىى & $r .19$ & 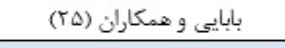 & $v$ \\
\hline CBM بر كاهش علادم اضطراب صفت كودكان با اختلال بيشقعالى و نقص توجه موثر است. & 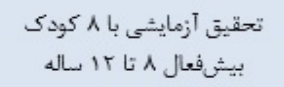 & $r \cdot 10$ & مير لو، قمرانى و راستى (T) & $\wedge$ \\
\hline سوكيرى شناختى در بهخاطر آوردن اطلاعات همخوان با خلق موثر است. & 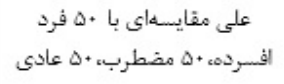 & $r \cdot 10$ & 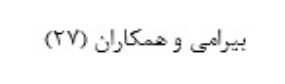 & 9 \\
\hline 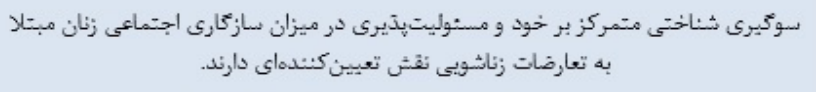 & روش همبستصنى & $r \cdot 10$ & ترخان و الحمدى (TA) & 1. \\
\hline
\end{tabular}

جدول r- خلاصه نتايج مطالعات خارجى انجام شده

\begin{tabular}{|c|c|c|c|c|}
\hline خلاصه نتايج & نوع تحقيق & 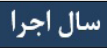 & 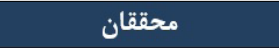 & رديف \\
\hline CBM بر كاهش اختلال اضطرابى نوجوانان مؤثر است. & تحقيق آزمايشى & T.T. & يتيت و همكاران (Y9) & 1 \\
\hline Pred ABM بر بيشبينى سوكيرىهاى بعدى موثر است. & تحقيق آزمايشى با ؟ · انفر بهصورت آنلاين & $r \cdot 19$ & كلادوين، موبيوس و بكر (·r) & r \\
\hline اصلاح سوكيرى توجه بر شيوه برخورد افراد با احساساتشان مؤثر است. & 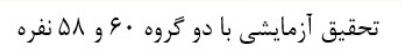 & $r .19$ & باك استال و همكاران (آ⿱艹) & r \\
\hline CBM بر كاهش اضطراب اجتماعى مؤثر است. & $\begin{array}{l}\text { تحقيق مداخلهاى در مورد } 19 \text { نوجوان با اضطراب اجتماعى بالا } \\
\text { بال }\end{array}$ & $r \cdot 1 \Lambda$ & ليشك و همكاران (rT) & p \\
\hline CBM بر كاهش شدت علاثم افسردىى مؤثر است. & تحقيق آزمايشى با وجا فرد افسرده بالينى & $r \cdot 1 \Lambda$ & ريجسن و همكاران (rT) & $\Delta$ \\
\hline CBT , CBM به اندازه در كاهش علاثم اضطراب اجتماعى مؤثر هستند. & تحقيق آزمايشى با بץ شركت كننده & $r \cdot 1 \Lambda$ & هوبرت و همكاران (TY) & 9 \\
\hline CBM در بِيشَّيرى از عود مصرف الكل موثر است. & تحقيق آزمايشى ه·fl| فرد معتاد به الكل & $r \cdot 1 \Lambda$ & رينك و همكاران (T山) & $\checkmark$ \\
\hline IBM بر كاهش سو & تحقيق نيمه آزمايشى. Frا دانشجو & $r \cdot 1 \Lambda$ & سالمينك و همكاران (ع) & $\wedge$ \\
\hline CBM بر كاهش آزردگى كمك خواهى براى درمان مؤثر است. & تحقيق نيمه آزمايشى با بr دانشجو & $r \cdot 1 \Lambda$ & استانلى، هم و جوينر (TV) & 9 \\
\hline CBM در مقابله با فراخوانى نشخوار افكار منفى موثر است. & تحقيق نيمه آزمايشى & $r \cdot I V$ & هرتل و همكاران (ז^) & 1. \\
\hline 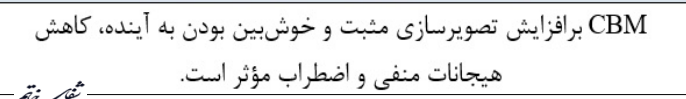 & تحقيق نيمه آزمايشى بال VV بزركسال & $r \cdot 10$ & مورفى و همكاران (T) & 11 \\
\hline
\end{tabular}




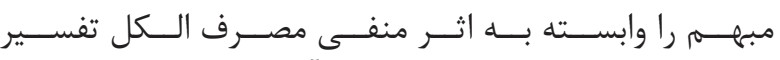

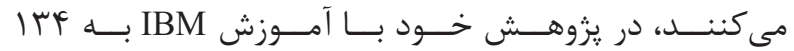

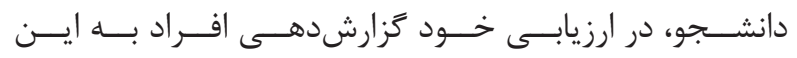

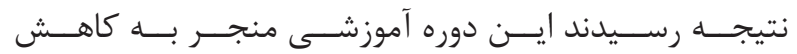

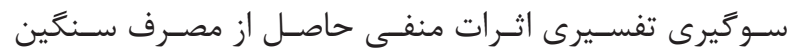

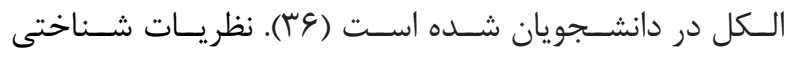

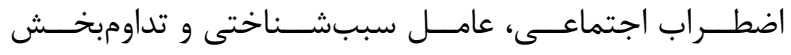

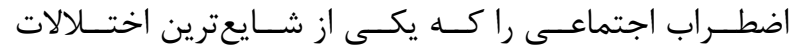

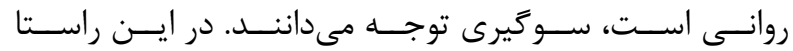

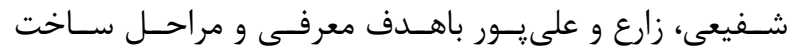

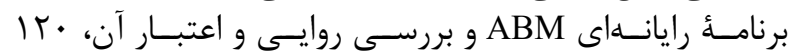

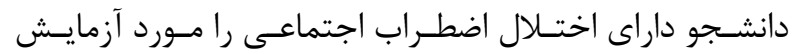

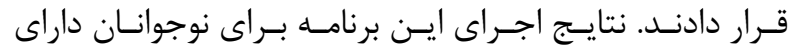

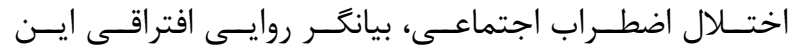

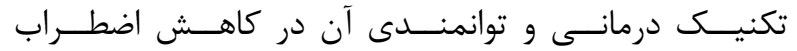

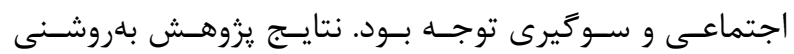

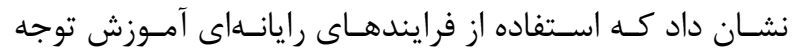

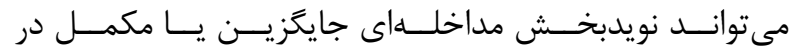

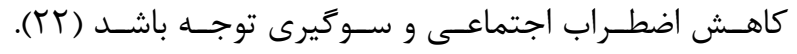

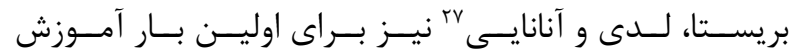

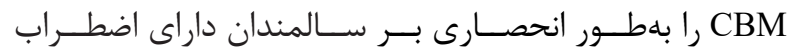

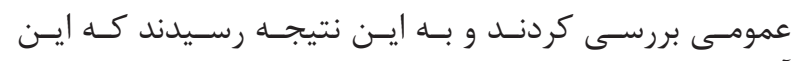

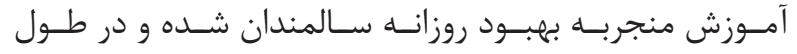

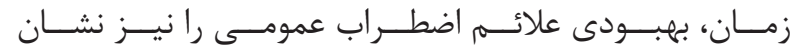

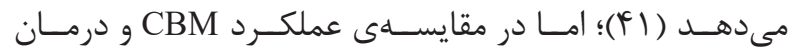

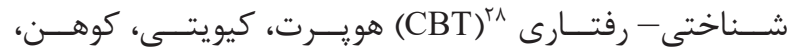

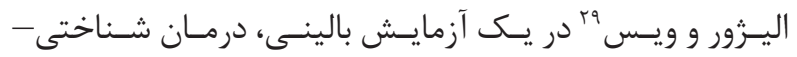

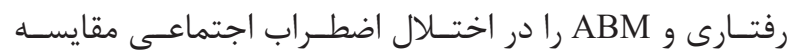

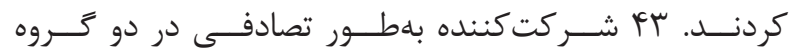

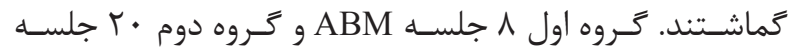

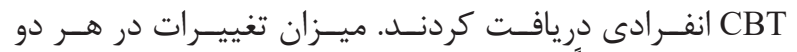

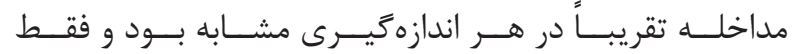

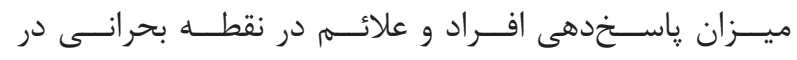

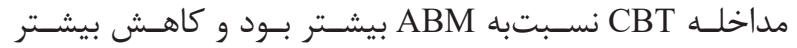

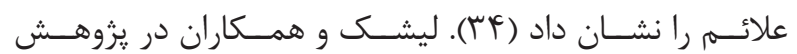

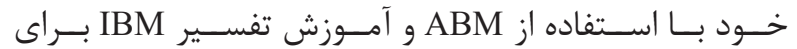

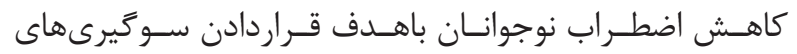

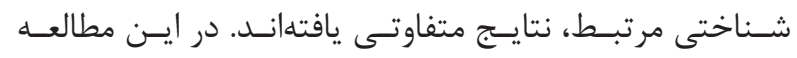

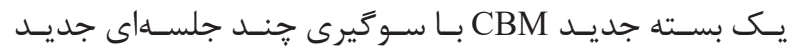

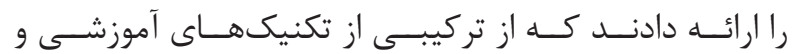

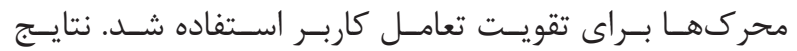

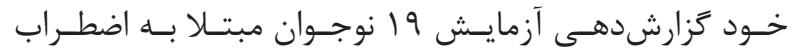

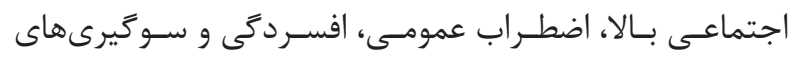

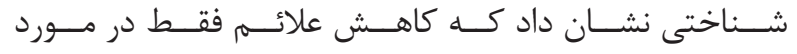

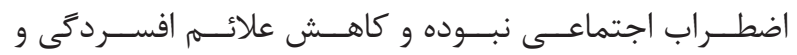

\footnotetext{
${ }^{24}$ MacLeod

${ }^{25}$ Murphy

${ }^{26}$ Salemink, Woud, Roos, Wiers \&Lindgren
}

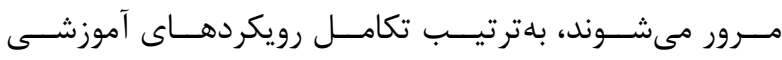

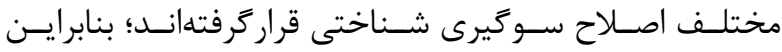

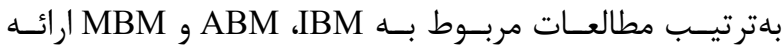

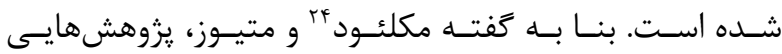

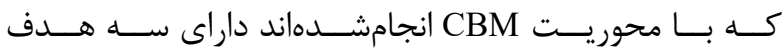

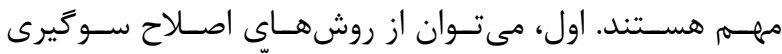

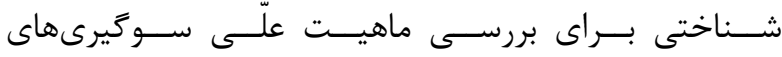

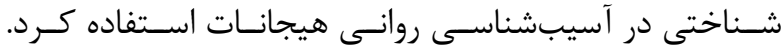

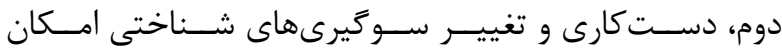

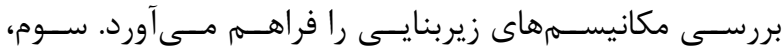

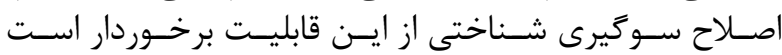

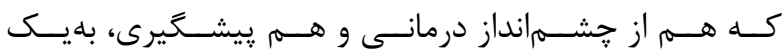

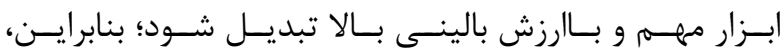

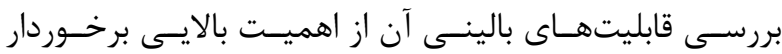

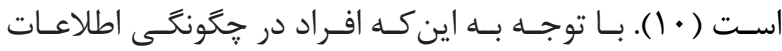

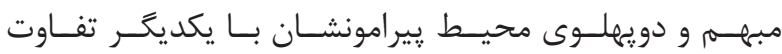

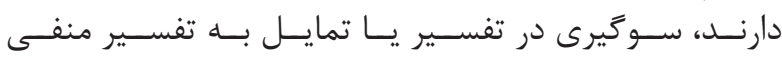

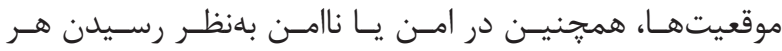

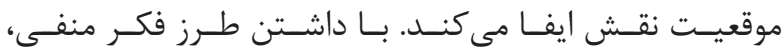

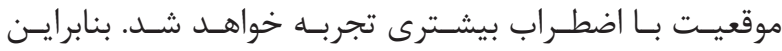

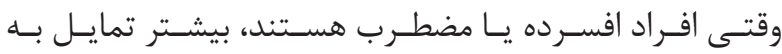

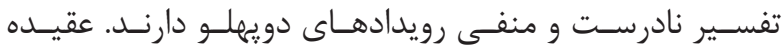

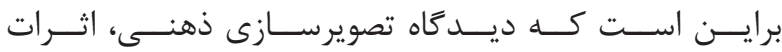

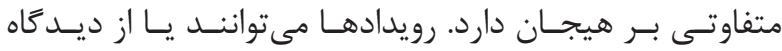

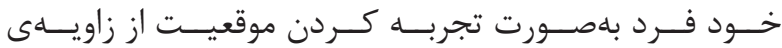

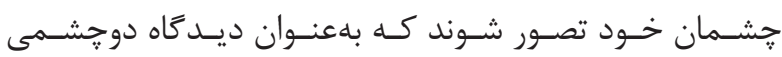

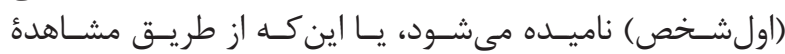

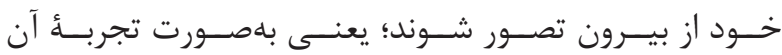

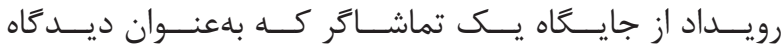

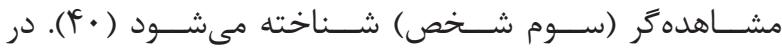

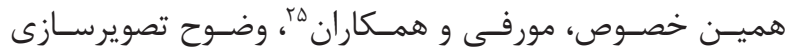

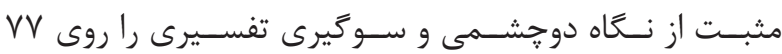

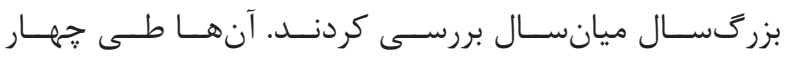

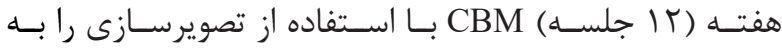

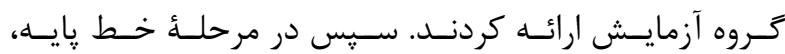

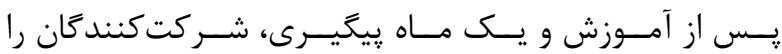

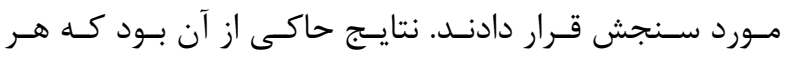

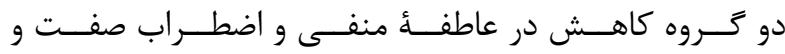

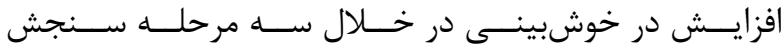

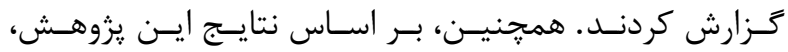
CBM

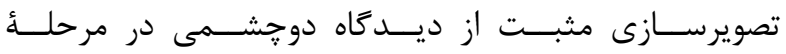

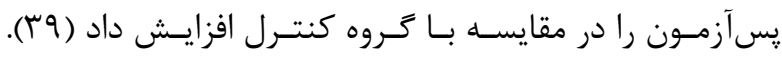

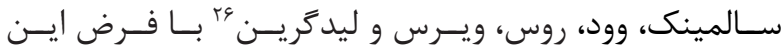

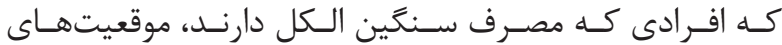

\footnotetext{
${ }^{27}$ Bristow, Leddy, Ononaiye

${ }^{28}$ Cogetive Behavior Therapy

${ }^{29}$ Huppert, Kivity, Cohen, Strauss, Elizur, Weiss
} 


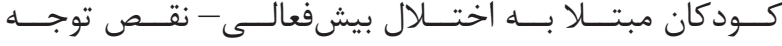

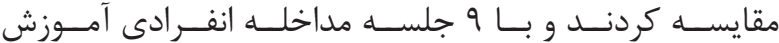

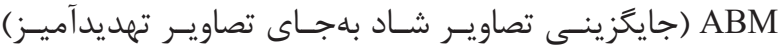

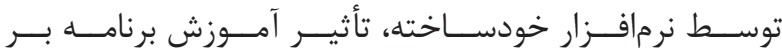

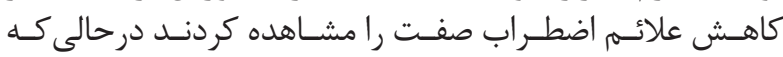

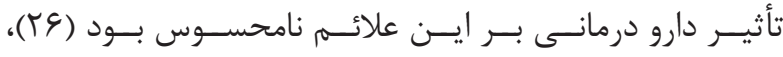

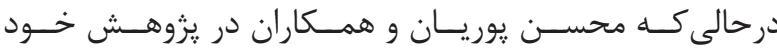

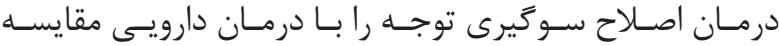

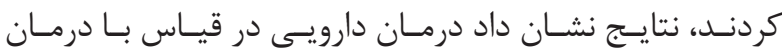

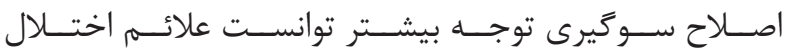

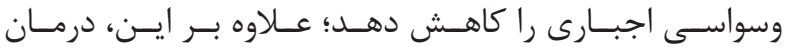

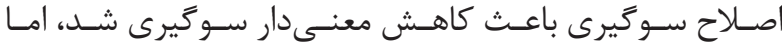

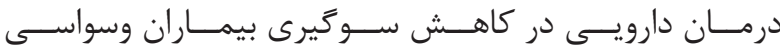

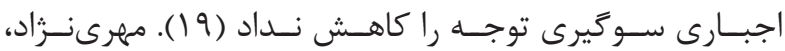

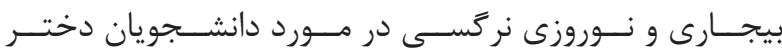

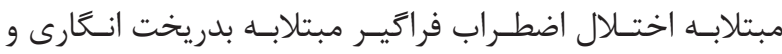

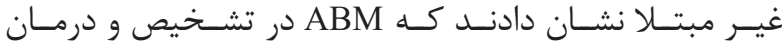

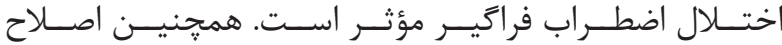

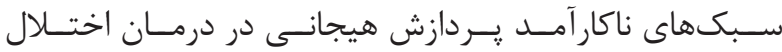

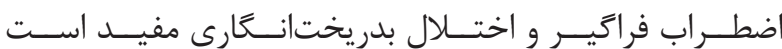

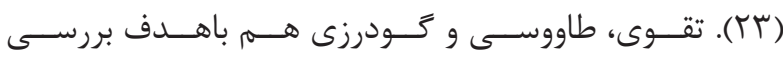

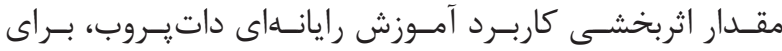

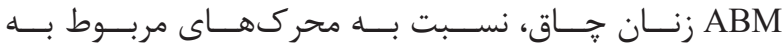

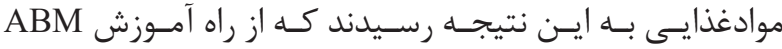

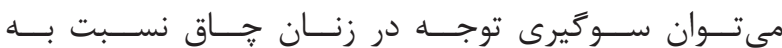

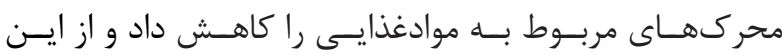

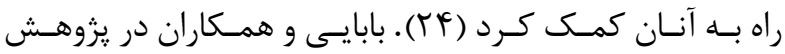

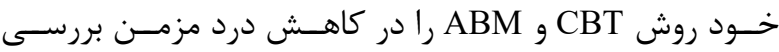

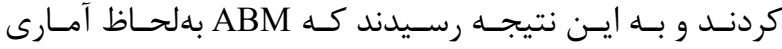

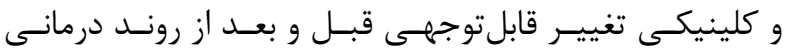

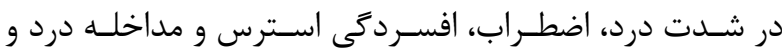

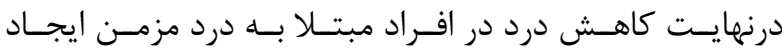

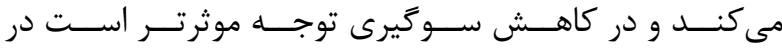

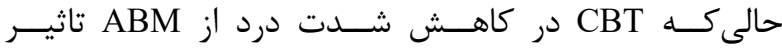

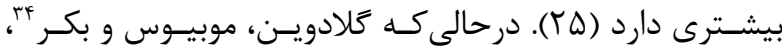
اصـاح سـو (Pred ABM)

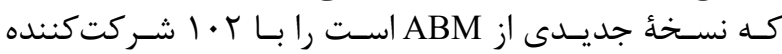

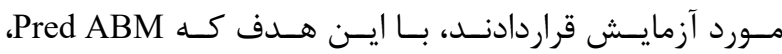

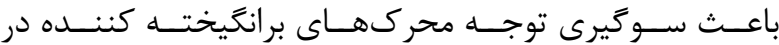

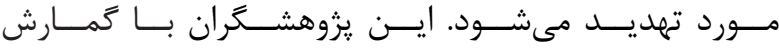

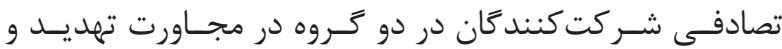

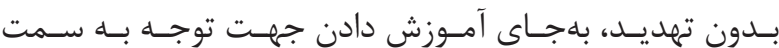

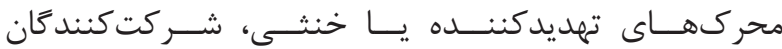

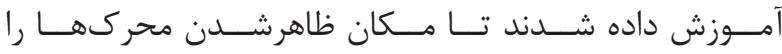

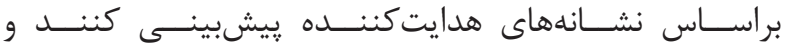

\footnotetext{
${ }^{30}$ Brown

${ }^{31}$ White

${ }^{32}$ Van Bockstaele
}

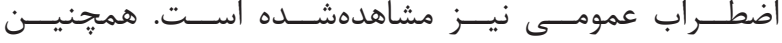

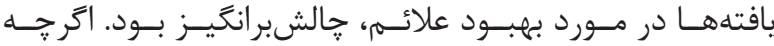

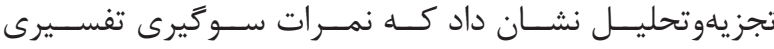

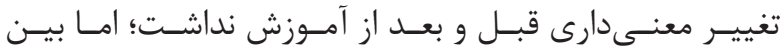

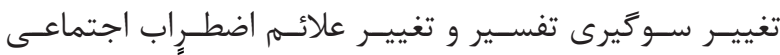

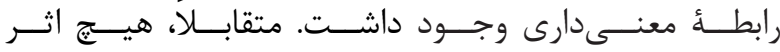

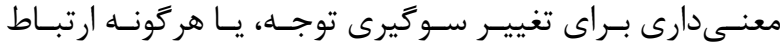

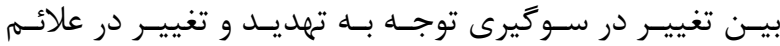

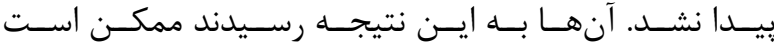

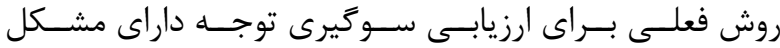

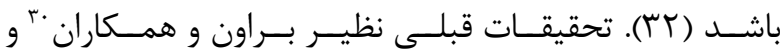

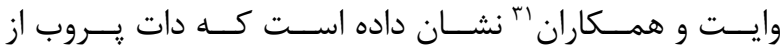

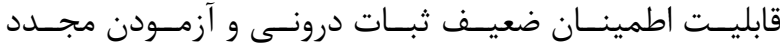

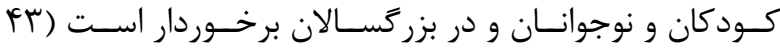

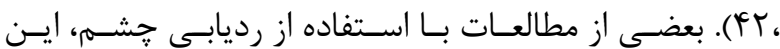

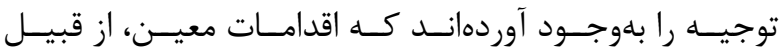

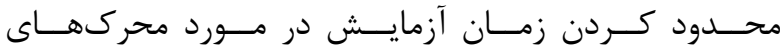

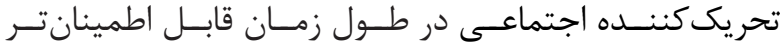

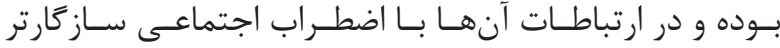

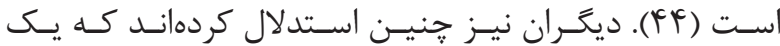

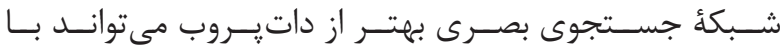

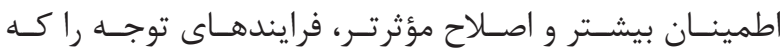

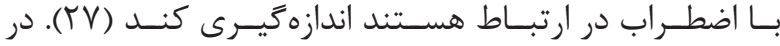

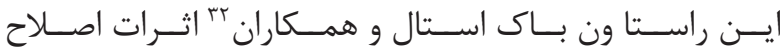

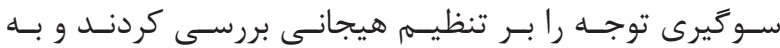

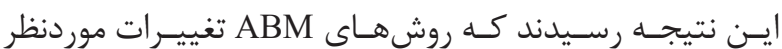

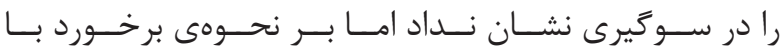

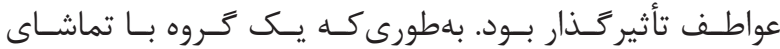

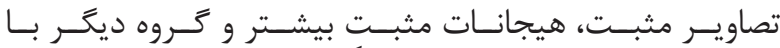

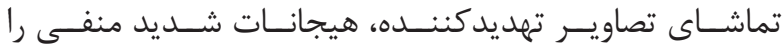

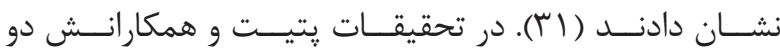

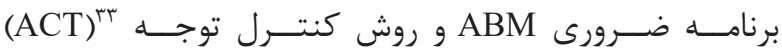

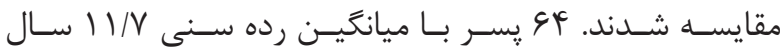

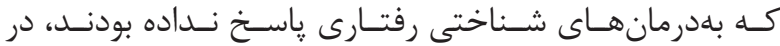

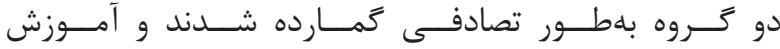

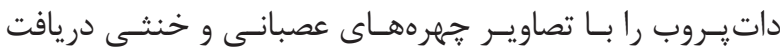

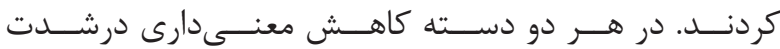

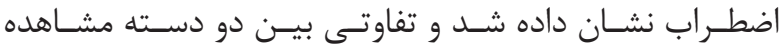

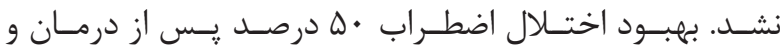

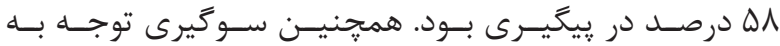

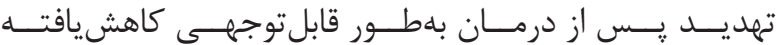

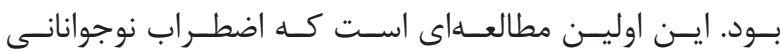

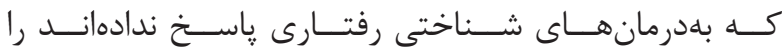

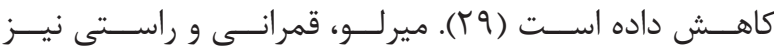

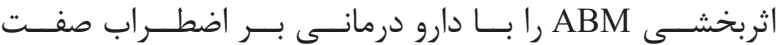

\footnotetext{
${ }^{33}$ Attention Control Trainin

${ }^{34}$ Gladwin, Möbius, Becker

${ }^{35}$ Predictive ABM
} 


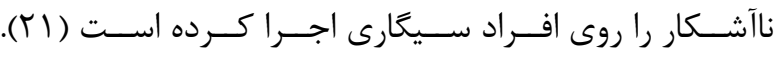

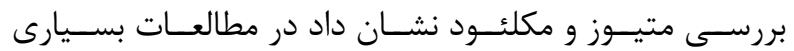

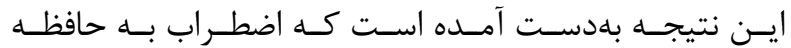

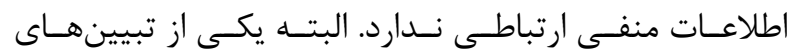

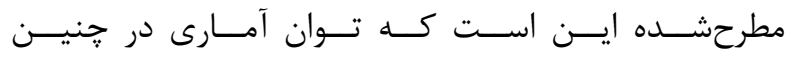

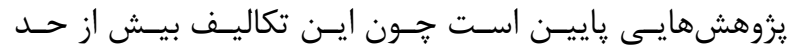

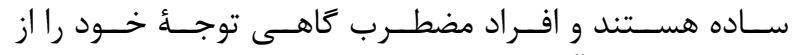

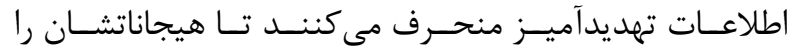

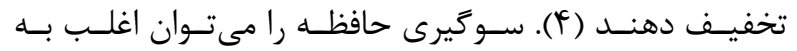

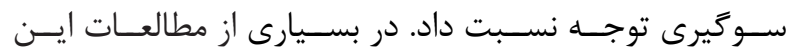

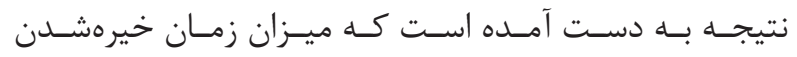

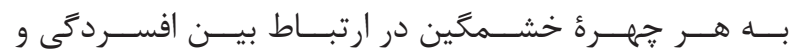

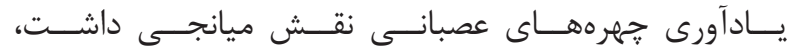

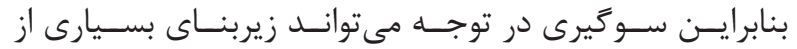

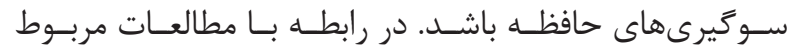

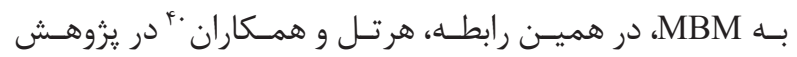

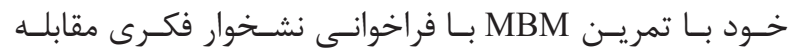

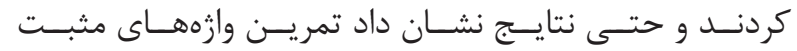

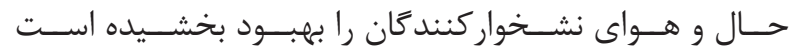

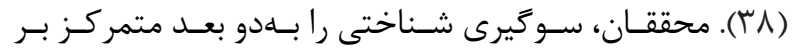

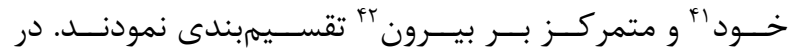

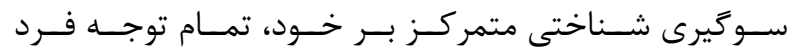

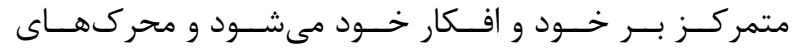

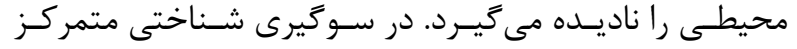

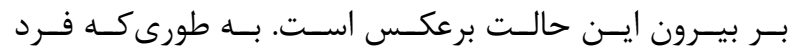

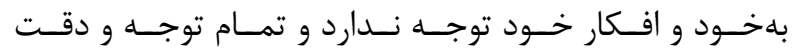

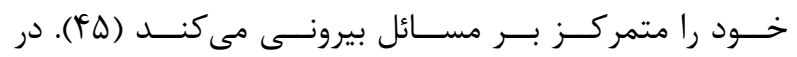

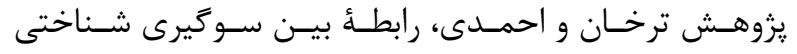

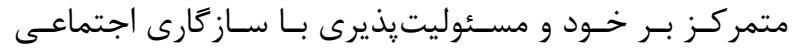

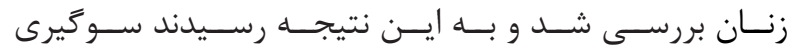

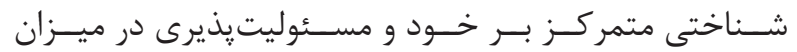

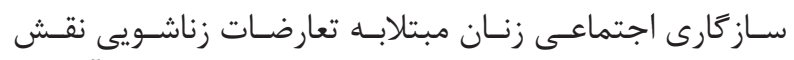

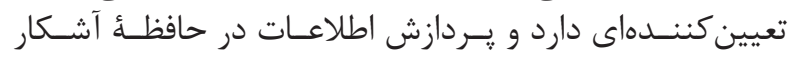

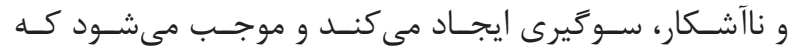

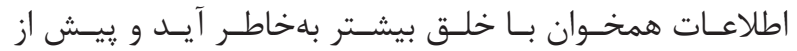

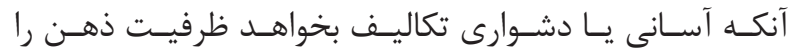

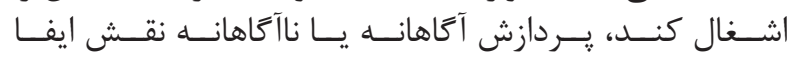

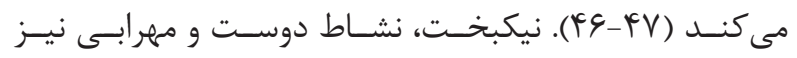

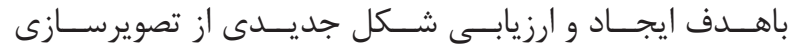

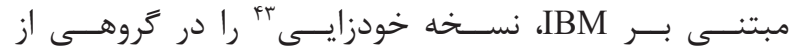

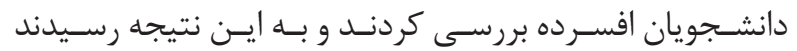

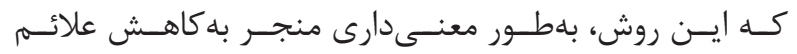

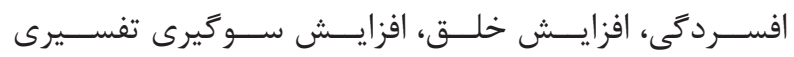

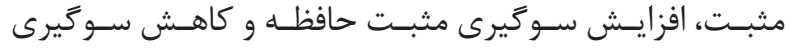

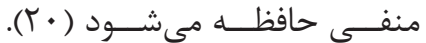

${ }^{36}$ Rinck, Wiers, Becker \& Lindenmeyer

${ }^{37}$ Stanly,Hom \& Joiner

${ }^{38}$ CBM- Help Seeking

${ }^{39}$ Approach Avoidance Task

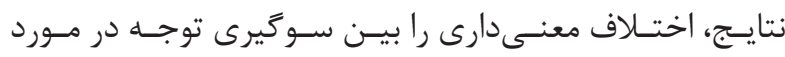

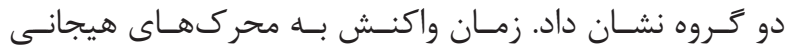

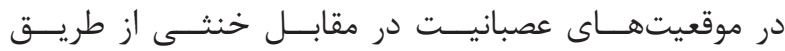

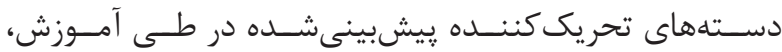

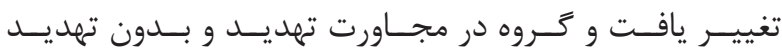

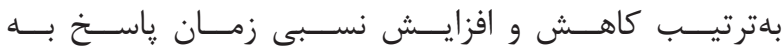

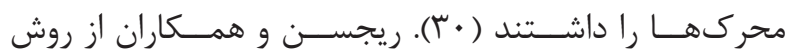
CBM

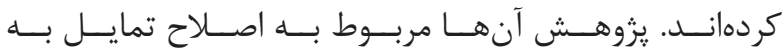

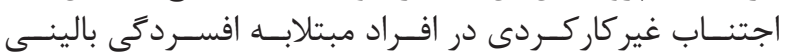

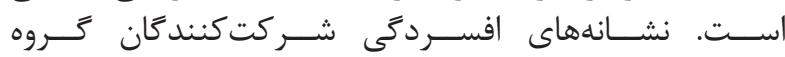

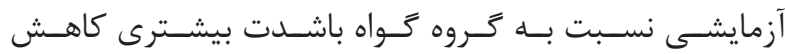

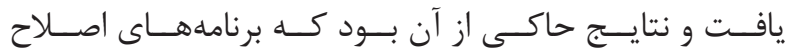

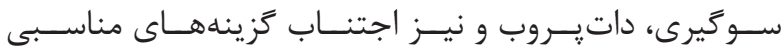

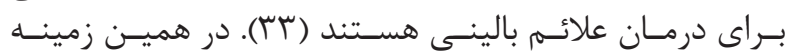

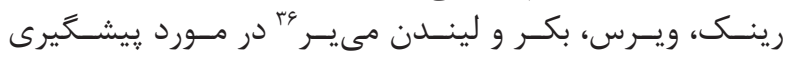

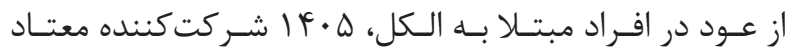

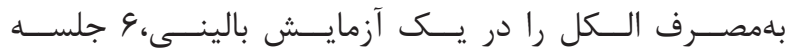

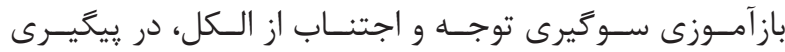

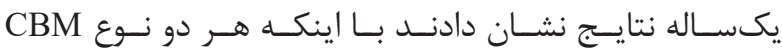

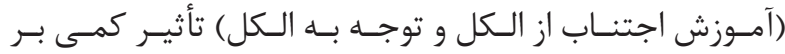

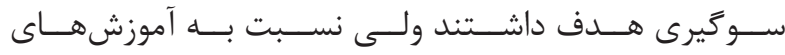

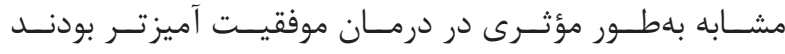

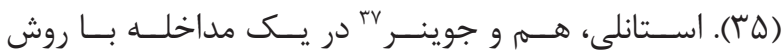

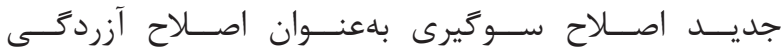

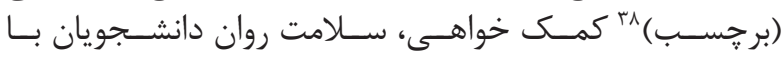

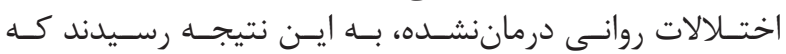

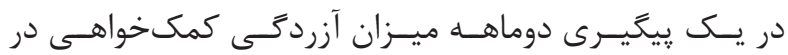

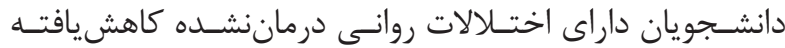

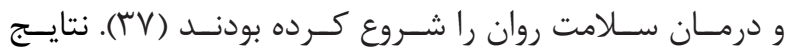

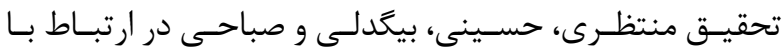

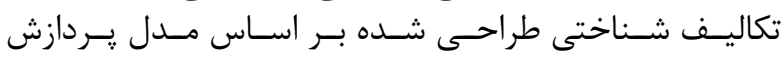

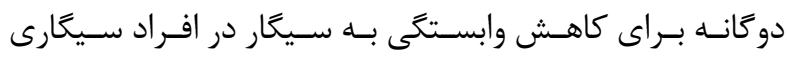

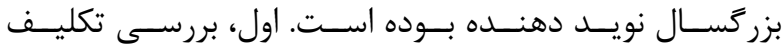

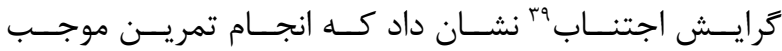

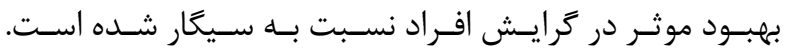

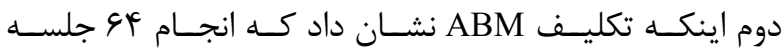

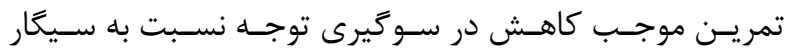

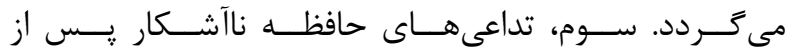

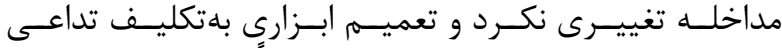

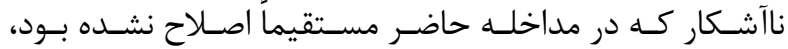

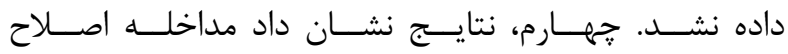

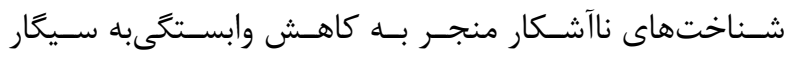

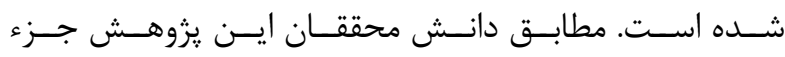

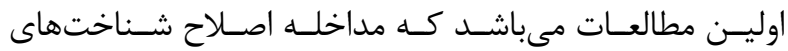

${ }^{40}$ Hertel

${ }^{41}$ Self- focus

${ }^{42}$ External Focus

${ }^{43}$ Self-generation 


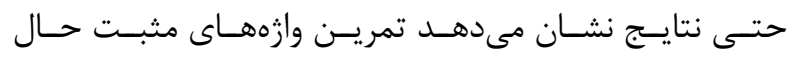

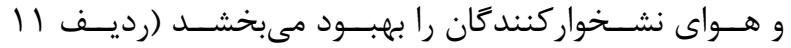

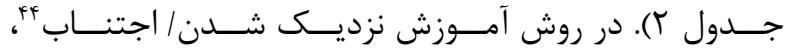

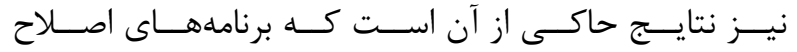

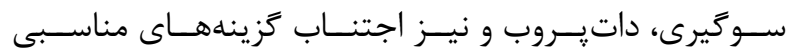

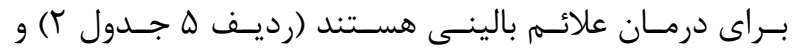

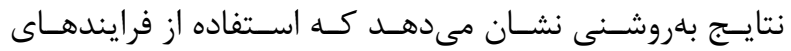

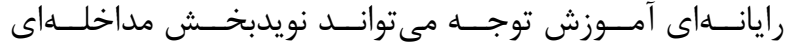

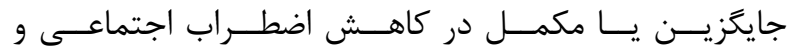

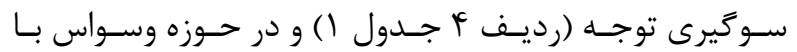

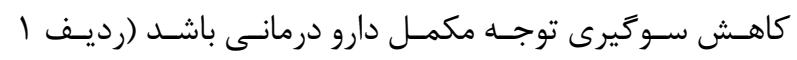

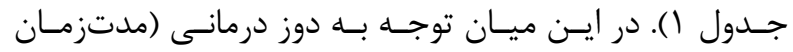

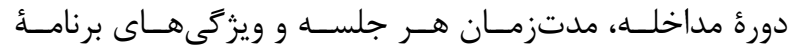

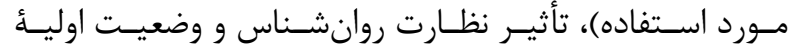

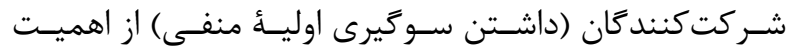

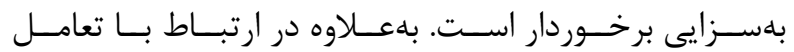

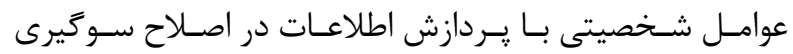

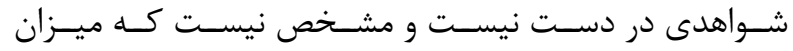

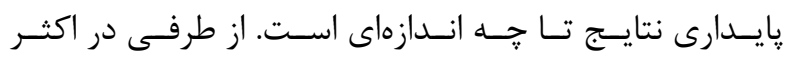

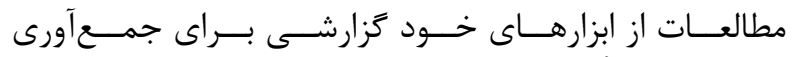

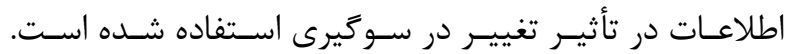

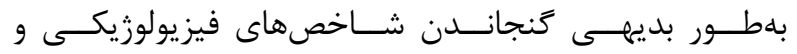

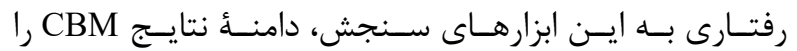

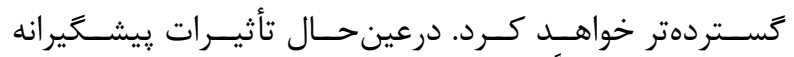

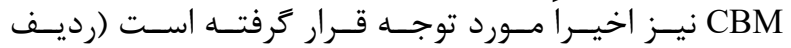

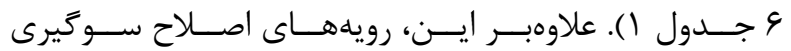

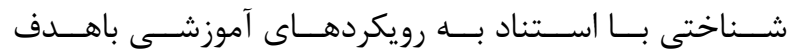

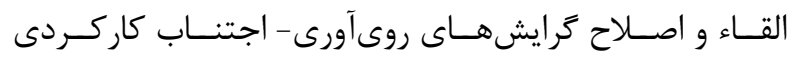

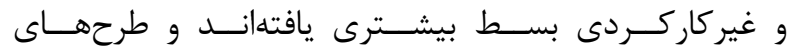

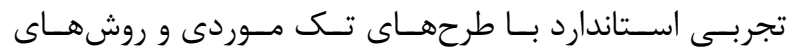

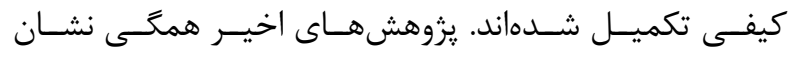

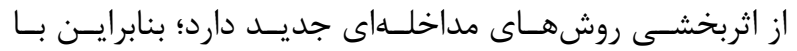

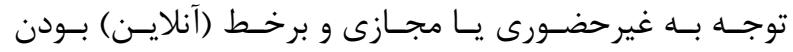

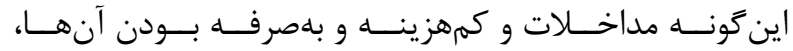

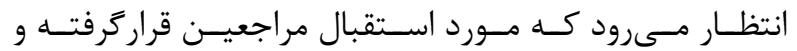

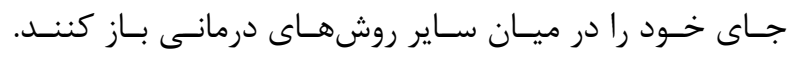

1. Mogg K, Millar N, Bradley BP. Biases in eye movements to threatening facial expressions in generalized anxiety disorder and depressive disorder. J abnorm psychol. 2000; 109(4): 695.

2. Clark DM, McManus F. Information processing in social phobia. Biologicalpsychiatry.2002;51(1):92-100.

3. Mayne T, Bonanno GA. Emotions: Current issues and future directions: Guilford Press; 2001.

$\begin{array}{lrrr}\begin{array}{l}\text { 4. Mathews A, } \\ \text { vulnerability }\end{array} \text { to } & \text { emotional } & \begin{array}{l}\text { Cognitive } \\ \text { disorders. }\end{array}\end{array}$

نتيجه

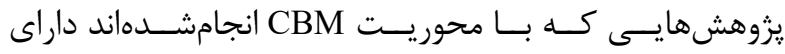

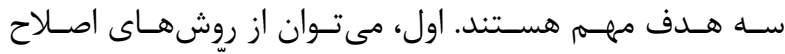

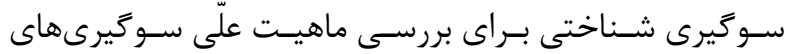

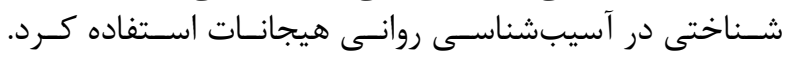

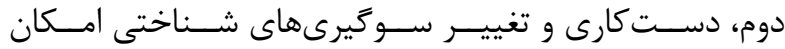

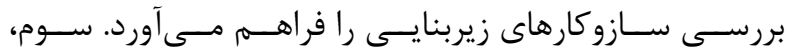

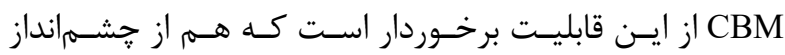

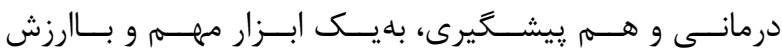

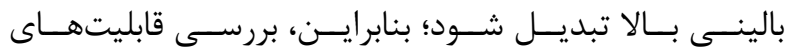

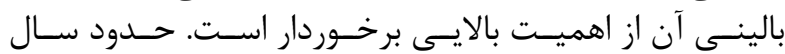

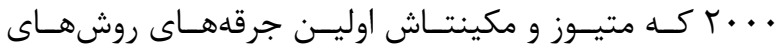

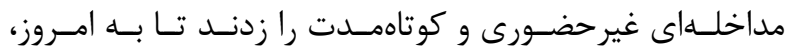

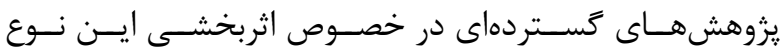

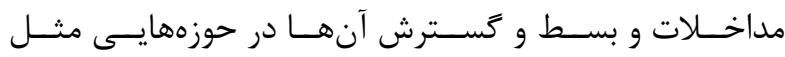

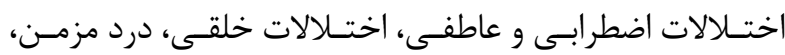

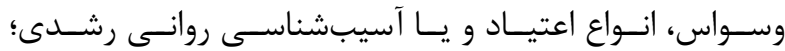

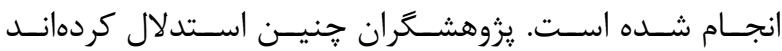

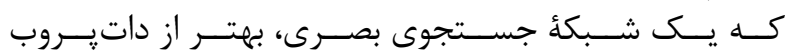

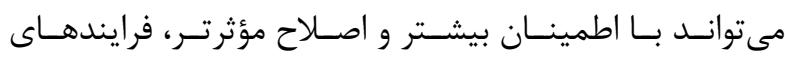

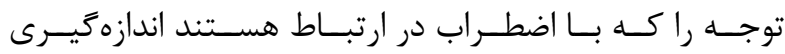

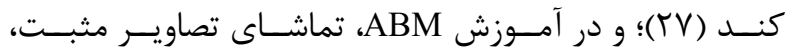

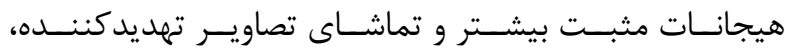

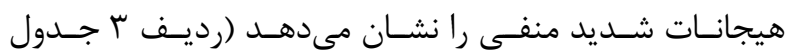

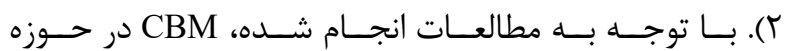

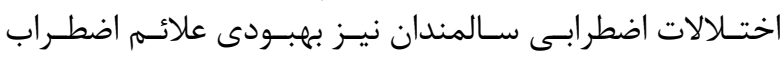

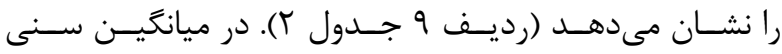

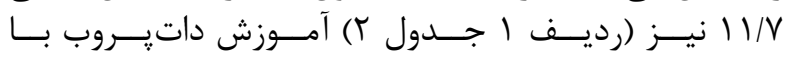

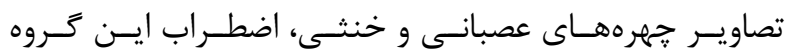

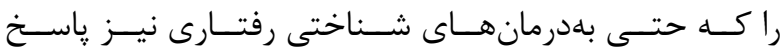

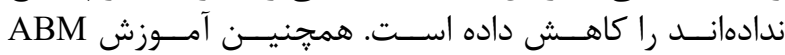

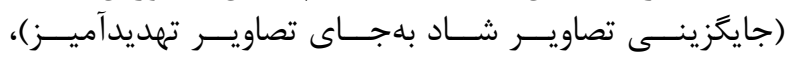

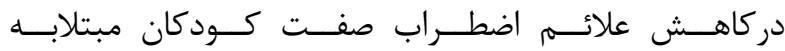

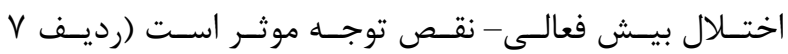

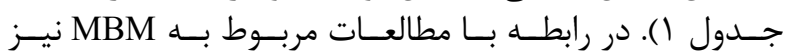

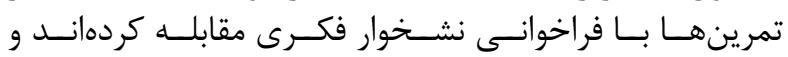

Annu Rev Clin Psychol. 2005; 1: 167-95.

5. Peckham AD, McHugh RK, Otto MW. A metaanalysis of the magnitude of biased attention in depression. Depress Anxiety. 2010; 27(12): 1135-42.

6. Peckham AD, McHugh RK, Otto MW. A meta-analysis of the magnitude of biased attention in depression. Depression and anxiety. 2010; 27(12): 1135-42.

7. Watkins ER, Baeyens CB, Read R. Concreteness training reduces dysphoria: proof-of-principle for repeated cognitive bias modification in depression. 
J Abnorm Psychol. 2009; 118(1): 55-64.

8. Papageorgiou C, Wells A. Effects of attention training on hypochondriasis: A brief case series. Psychol Med. 1998; 28(1): 193-200.

9. Mathews A, Ridgeway V, Cook E, Yiend J. Inducing a benign interpretational bias reduces trait anxiety. J behav Ther Exp Psychiatry. 2007; 38(2): 225-36.

10. Burack JA, Enns JT, Fox NA. Cognitive Science, Development, and Psychopathology: Typical and Atypical Developmental Trajectories of Attention: O U P; 2012.

11. MacLeod C, Mathews A. Cognitive bias modification approaches to anxiety. Annu Rev Clin Psychol. 2012; 8: 189-217.

12. Browning $\mathrm{M}$, Holmes EA, Charles M, Cowen PJ, Harmer CJ. Using attentional bias modification as a cognitive vaccine against depression. Biol Psychiatry. 2012; 72(7): 572-9.

13. Mathews A, Mackintosh B. Induced emotional interpretation bias and anxiety. J Abnorm Psychol. 2000; 109(4): 602.

14. Eysenck MW, Mogg K, May J, Richards A, Mathews A. Bias in interpretation of ambiguous sentences related to threat in anxiety. J Abnorm Psychol. 1991; 100(2): 144.

15. Khodadadi M, Amani H. Semantic Stroop Task. Tehran: Sina Institute of Cognitive Behavioral Sciences Research 2014.

16. Khodadadi M, Feyzi MR, Movahedi Y, Ahmadi I. Assessment of attention bias in the cognitive processing of neutral and emotional words Using semantic Strop test. J Cogn Psychol Psychiatry. 2014; 1(1): 23-30.

17. Khodadadi M, Nokani M, Amani H. Dot probe task and cognitive bias modification software. Institute for behavioral and cognitive sciences. Tehran: Sina Institute of Cognitive Behavioral Sciences Research; 2015.

18. Yiend J, Lee J-S, Tekes S, Atkins L, Mathews A, Vrinten M, et al. Modifying interpretation in a clinically depressed sample using 'cognitive bias modification-errors': A double blind randomised controlled trial. Cognit Ther Res. 2014; 38(2): 146-59.

19. Mohsenpoorian Kh, Nokani M, Jamilian H, Ghasemi M. Comparison of Attention Bias Modification With Selective Serotonin Reuptake Inhibitors in Patients With Obsessive-Compulsive Disorder. J Arak Uni Med Sci. 2019; 22(4): 76-85.
20. Nikbakht A, Neshat Doust HT, Mehrabi H. Effect of positive imagery-based interpretation bias modification, interpretation bias modification based self-generation and computerized cognitivebehaviour training on depressed students. 2018.

21. Montazeri M.S MHS, Bigdeli I, Sabahi P. Effectiveness of Therapeutic Intervention of Cognitive Bias Modification (CBM) on Cigarette Dependency. J Psychol Achiev 2017; 24(2): 1-22.

22. Shafiei h, Zare H, Alipor A. Construction and Validation of Computer Program of Attention Bias Modification Treatment for Social Anxiety Disorder. Adv Cogn Psychol 2017; 19(2): 19-29.

23. Mehrinejad SA, Farah Bijari A, Norouzi NM. Comparison of attention bias and emotional processing styles in female students with generalized anxiety disorder, with body dysmorphic disorder and inpatient. Clin Psychol Sci 2016; 6(34): 99-114.

24. Taghavi M, Tavoosi A, Goudarzi M. The Effect of Attentional Bias Modification Towards Food Cues on Attention of Obese Women. Psychol. Methods. Models. 2016; 7(25): 1-10.

25. Babai F, Sepavand T, Nokani M, Aghamohammadi M, Sheybani F. Effectiveness of attentional bias modification and cognitive behavioral therapy on the reduction of pain intensity in patients with chronic pain. Int J Med Res Health Sci. 2016; 5(8): 273-8.

26. Mirloo S, GHomrani A, Rasti J. Comparison of the effect of attention bias modification with pharmacotherapy on anxiety traits in children ADHD. Contemp Psychol 2015; 10, 1580-1582.

27. Beyrami M, Movahedi Y, Poormohammadi M, Ahmadi L. Cognitive Bias in Emotional Information Processing in Anxious, Depressed and Normal People. J Cogn Psychol. 2015; 2(4).

28. Tarkhan M, Ahmadi LG.The Relationship Between Self-focus Cognitive Bias and Responsibility with Social Adjustment of Women. Soc Cogn 2015; 3: 97-108.

29. Pettit JW, Bechor M, Rey Y, Vasey MW, Abend $\mathrm{R}$, Pine DS, et al. A randomized controlled trial of attention bias modification treatment in youth with treatment-resistant anxiety disorders. J Am Acad Child Adolesc Psychiatry. 2020; 59(1): 157-65.

30. Gladwin TE, Möbius MM, Becker ES. Predictive attentional bias modification induces stimulus-evoked attentional bias for threat. Eur J Psychol. 2019; 15(3): 479-90. 
31. Van Bockstaele B, Notebaert L, Macleod C, Salemink E, Clarke PJ, Verschuere B, et al. The effects of attentional bias modification on emotion regulation. J Behav Ther Exp Psychiatry. 2019; 62: 38-48.

32. Lisk SC, Pile V, Haller SP, Kumari V, Lau JY. Multisession cognitive bias modification targeting multiple biases in adolescents with elevated social anxiety. Cognit Ther Res. 2018; 42(5): 581-97.

33. Vrijsen JN, Fischer VS, Müller BW, Scherbaum N, Becker ES, Rinck M, et al. Cognitive bias modification as an add-on treatment in clinical depression: Results from a placebo-controlled, single-blinded randomized control trial. J Affect Disord 2018; 238: 342-50.

34. Huppert JD, Kivity Y, Cohen L, Strauss AY, Elizur Y, Weiss M. A pilot randomized clinical trial of cognitive behavioral therapy versus attentional bias modification for social anxiety disorder: an examination of outcomes and theory-based mechanisms. J Anxiety Disord. 2018; 59: 1-9.

35. Rinck M, Wiers RW, Becker ES, Lindenmeyer J. Relapse prevention in abstinent alcoholics by cognitive bias modification: Clinical effects of combining approach bias modification and attention bias modification. J Consult Clin Psychol. 2018; 86(12): 1005.

36. Salemink E, Woud ML, Roos M, Wiers R, Lindgren KP. Reducing alcohol-related interpretive bias in negative affect situations: Using a scenariobased Cognitive Bias Modification training paradigm. Addict Behav. 2019; 88: 106-13.

37. Stanley IH, Hom MA, Joiner TE. Modifying mental health help-seeking stigma among undergraduates with untreated psychiatric disorders: A pilot randomized trial of a novel cognitive bias modification intervention. Behav Res Ther. 2018; 103: 33-42.

38. Hertel PT, Maydon A, Cottle J, Vrijsen JN. Cognitive bias modification: Retrieval practice to simulate and oppose ruminative memory biases. Clin Psychol Sci. 2017; 5(1): 122-30.
39. Murphy SE, O'Donoghue MC, Drazich EH, Blackwell SE, Nobre AC, Holmes EA. Imagining a brighter future: The effect of positive imagery training on mood, prospective mental imagery and emotional bias in older adults. Psychiatry Res. 2015; 230(1): 36-43.

40. Holmes EA, Lang TJ, Shah DM. Developing interpretation bias modification as a" cognitive vaccine" for depressed mood: imagining positive events makes you feel better than thinking about them verbally. J Abnorm Psychol. 2009; 118(1): 76.

41. Bristow K.E LA, Ononaiye M. The influence of interpretive $\mathrm{CBM}$ on generalized anxiety in older adults. PhD thesis Univ East Anglia. 2017.

42. Van Bockstaele B, Salemink E, Bögels SM, Wiers RW. Limited generalisation of changes in attentional bias following attentional bias modification with the visual probe task. Cogn Emot. 2017; 31(2): 369-76.

43. White LK, Britton JC, Sequeira S, Ronkin EG, Chen G, Bar-Haim Y, et al. Behavioral and neural stability of attention bias to threat in healthy adolescents. Neuroimage. 2016; 136: 84-93.

44. Lazarov A, Pine DS, Bar-Haim Y. Gazecontingent music reward therapy for social anxiety disorder: A randomized controlled trial. Am J Psychiatry. 2017; 174(7): 649-56.

45. Spector IP, Pecknold JC, Libman E. Selective attentional bias related to the noticeability aspect of anxiety symptoms in generalized social phobia. J Anxiety Disord. 2003; 17(5): 517-31.

46. Tarkhan M ALG-. The Relationship Between Selffocus Cognitive Bias and Responsibility with Social Adjustment of Women. Soc Cogn. 2015; 3: 97-108.

47. Beyrami M, Movahedi Y, Poormohammadi M, Ahmadi L. Cognitive Bias in Emotional Information Processing in Anxious, Depressed and Normal People. J Cogn Psychol. 2015; 2(4). 